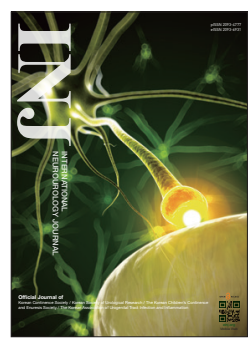

\title{
Implantable Devices for Sustained, Intravesical Drug Delivery
}

\author{
Seung Ho Lee ${ }^{1}$, Young Bin Choy ${ }^{1,2,3}$ \\ ${ }^{1}$ Institute of Medical \& Biological Engineering, Medical Research Center, Seoul National University, Seoul, Korea \\ ${ }^{2}$ Department of Biomedical Engineering, Seoul National University College of Medicine, Seoul, Korea \\ ${ }^{3}$ Interdisciplinary Program in Bioengineering, College of Engineering, Seoul National University, Seoul, Korea
}

\begin{abstract}
In clinical settings, intravesical instillation of a drug bolus is often performed for the treatment of bladder diseases. However, it requires repeated instillations to extend drug efficacy, which may result in poor patient compliance. To alleviate this challenge, implantable devices have been developed for the purpose of sustained, intravesical drug delivery. In this review, we briefly summarize the current trend in the development of intravesical drug-delivery devices. We also introduce the most recently developed devices with strong potential for intravesical drug-delivery applications.

Keywords: Urinary Bladder Disease; Drug Delivery System; Intravesical Drug Delivery; Sustained Drug Delivery

- Grant Support: This work was supported by a grant from the Korea Healthcare Technology R\&D Project, Ministry for Health, Welfare \& Family Affairs, Republic of Korea (HI14C2194) and by a grant of the Bio \& Medical Technology Development Program of the National Research

Foundation funded by the Ministry of Science, ICT \& Future Planning (2015M3A9E2030129).

- Conflict of Interest: No potential conflict of interest relevant to this article was reported.
\end{abstract}

\section{INTRODUCTION}

Bladder diseases, such as overactive bladder (OAB), and interstitial cystitis/painful bladder syndrome (IC/PBS), have generated public health concern [1]. OAB, a chronic bladder disorder and debilitating condition of the lower urinary tract, negatively impacts the life-quality of millions of patients worldwide [2]. In the United States, the estimated prevalence of IC/PBS, a chronic bladder disease with unpleasant sensations, such as, pain, pressure, and discomfort, is 3-8 million women and 1-4 million men, with increasing occurrence with age [3].

In general, drug therapy is one of the most accepted modalities for the treatment of bladder diseases. A variety of drug formulations have been developed for oral administration $[4,5]$; however, oral medication may have low therapeutic benefit due to its poor systemic absorption and extensive first-pass liver metabolism, resulting in inefficiency in local bladder treatment [6,7]. In this sense, intravesical instillation of therapeutic agents is an attractive alternative treatment for bladder diseases and has long been studied in both academia and industry [8]. For intravesical drug installation, a determined amount of a bolus drug solution is infused into the bladder through a urethral catheter. In this way, a therapeutically effective concentration of drug can be achieved at the local site of interest, even with a lower drug dose, which can reduce possible systemic toxicity and side effects [9]. For example, it has been reported that a daily 1-hour instillation of lidocaine for 5 consecutive days could substantially relieve the symptoms of IC/PBS [10].

However, to be effective, this strategy requires repeated intravesical drug administrations with multiple catheter insertions into the urinary tract, and this relatively invasive procedure can cause discomfort and possibly even infection. Additionally, the

Corresponding author: Young Bin Choy (iD http://orcid.org/0000-0001-6300-9377 Department of Biomedical Engineering, Seoul National University College of Medicine, 103 Daehak-ro, Jongno-gu, Seoul 03080, Korea

E-mail: ybchoy@snu.ac.kr / Tel: +82-2-740-8592 / Fax: +82-2-741-6303

Submitted: June 7, 2016 / Accepted after revision: June 15, 2016

C) (i) This is an Open Access article distributed under the terms of the Creative Commons Attribution Non-Commercial License (http://creativecommons.org/licenses/by-nc/4.0/) which permits unrestricted non-commercial use, distribution, and reproduction in any medium, provided the original work is properly cited. 
majority of the instilled drug is easily eliminated via urination or diluted by urine production [11], which lowers drug bioavailability in the local bladder. To resolve this, there is a significant need for an intravesical device for sustained drug delivery. With a single insertion into the bladder, the device could release drug for a prolonged period of time, thereby minimizing the number of intravesical instillations. In this mini-review, we describe some work on drug-delivery devices for intravesical applications. We also introduce some other types of devices with technologies applicable to intravesical drug delivery to gain insight into future study endeavors in this area.

\section{DRUG-DELIVERY DEVICES DEVELOPED FOR INTRAVESICAL APPLICATIONS}

The intravesical devices need to meet the following design criteria. The device needs to be easily inserted into the bladder via the urinary tract. After insertion, the device needs to stay in the bladder without being easily expelled, e.g., via urination. Most importantly, while staying in the bladder, the device must release drug in a sustained manner for a prolonged period of time. Therefore, the intravesical devices currently under development are mostly in the shape of tubes that can be deformed to a more complicated shape after bladder insertion. The devices also possess a drug reservoir chamber and a proper barrier for slow drug release for a long time.
The UROS bladder pump (Situs Corp., San Diego, CA, USA) was initially presented at the 95th American Urological Association Meeting in 2000 [12]. The UROS pump consists of a drug reservoir and a pressure-responsive valve for controlling the drug release rate. A septum is located at the proximal end of the device to allow filling of the drug solution in the reservoir. A thread is assembled at the distal end for easy device extraction after drug release is completed. Thus, the pump is initially in a straight tube shape with a length of approximately $10 \mathrm{~cm}$ and an outer diameter of approximately $0.6 \mathrm{~cm}$ and thus, it can be inserted into the bladder using a cystoscope. The drug solution is infused into the device via the septum, which changes the device shape into a crescent shape, allowing its retention in the bladder. The UROS system was evaluated in clinical studies involving a total of 16 female subjects. The UROS filled with saline was inserted into thirteen healthy female subjects for three days, and they showed no side effects, indicating biocompatibility of the device. Three other female subjects diagnosed with $\mathrm{OAB}$ received the UROS loaded with oxybutynin, an anticholinergic agent that is known to block bladder contraction [13], for 24 hours, and the results showed acceptable tolerance levels and no side effects [12].

An intravesical device for lidocaine delivery was introduced by Lee and Cima [14]. This device is also fabricated in a tube shape; however, it is designed to be prefilled with drug. The shape change after bladder insertion occurs via shape-memory
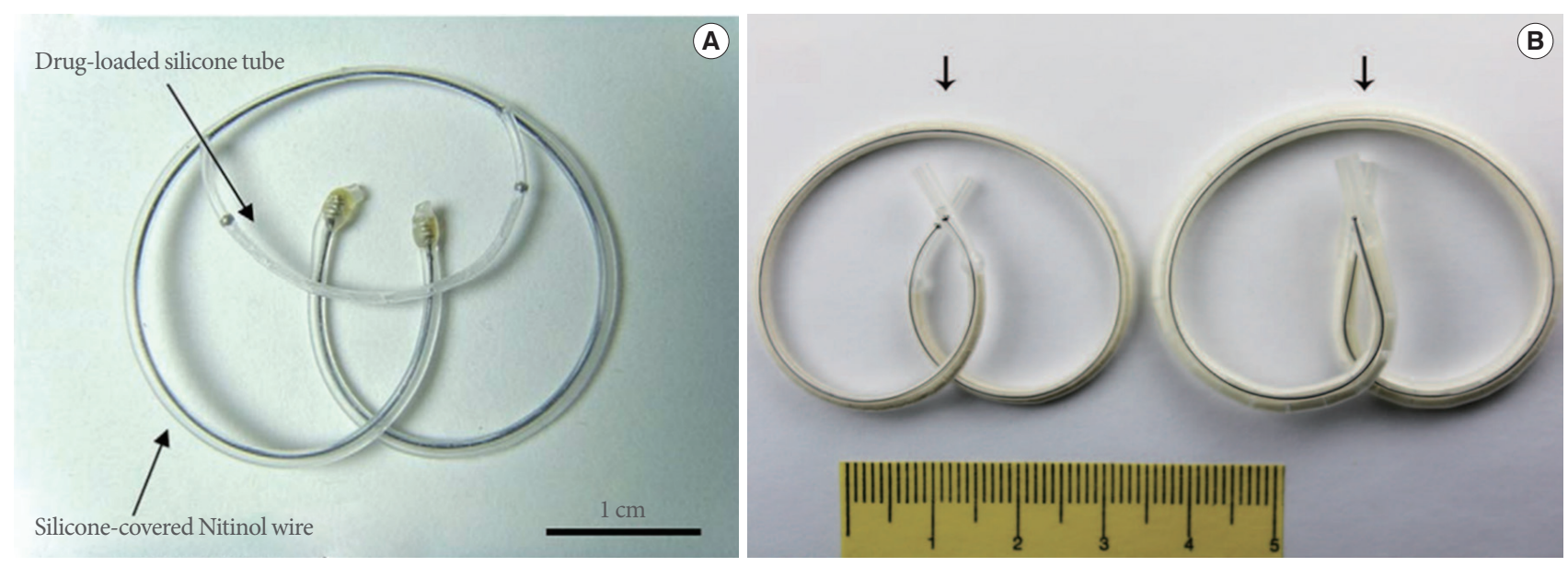

Fig. 1. Devices for intravesical lidocaine delivery. The device is assembled with a superelastic shape-memory wire for both easy insertion and better retention in the bladder. (A) Prototype device for preclinical study. Adapted from Lee H, et al. J Control Release 2011;149:133-9, with permission of American Association for the Advancement of Science [14]. (B) LiRIS (TARIS Biomedical, Lexington, MA, USA) for clinical study, where the arrows indicate the locations of the laser-drilled orifices. The ruler is $5 \mathrm{~cm}$ long. Adapted from Nickel JC, et al. Sci Transl Med 2012;4:143ra100, with permission of Elsevier [15]. 
wire in the device. As a prototype (Fig. 1A), the device was made of a silicone tube (inside diameter [I.D.] $\times$ outside diameter [O.D.]: $305 \mu \mathrm{m} \times 635 \mu \mathrm{m}$ ), which served as drug reservoir filled with lidocaine salt crystals. Along this tube, an additional silicone tube (I.D. $\times$ O.D.: $508 \mu \mathrm{m} \times 940 \mu \mathrm{m}$ ) filled with a shapememory wire made of nitinol was assembled. Thus, the device can be straightened while being inserted via the urinary tract into the bladder and is then bent into a pretzel-like shape by the superelasticity of the shape-memory wire. In the bladder, the aqueous fluid from the urine diffuses into the drug reservoir via the semipermeable silicone tube wall to dissolve lidocaine salt, which in turn, builds up an osmotic pressure to push lidocaine via the small laser-drilled orifice, $50 \mu \mathrm{m}$ in diameter, formed in the middle of the silicone drug reservoir tube. When tested in living rabbit models, therefore, the device could release the drug at a nominal rate of $49.51 \mu \mathrm{g} / \mathrm{hr}$ for 12 hours. Based on this technique, the LiRIS (i.e., Lidocaine-Releasing Intravesical System, TARIS Biomedical, Lexington, MA, USA) was recently developed and is currently under a phase 1 safety-assessment trial for the site-specific treatment of IC/PBS (Fig. 1B). The device is loaded with either 200 or $650 \mathrm{mg}$ of lidocaine and is de- signed to provide an initial zero-order release for the first 24 hours, followed by primary first-order release throughout the remaining 14 days [15].

\section{DRUG-DELIVERY DEVICES FOR POSSIBLE INTRAVESICAL APPLICATIONS}

Although not originally purposed for intravesical applications, there is a variety of devices with strong potential for intravesical drug delivery. One example is the devices for intrauterine drug delivery, which also consider ease of insertion, device retention in the target area and sustained, local drug release. The Progestasert (Alza, Mountain View, CA, USA) device consists of a Tshaped polyethylene (PE) vinyl acetate frame, where a vertical cylinder in a "T" shape, $36 \mathrm{~mm}$ in length, is a drug reservoir filled with a contraceptive drug, progesterone (Fig. 2A) [16]. The flexible T-frame ( $32 \mathrm{~mm}$ in length) can be folded during insertion and can be unfolded in the uterus to retain the device in the local insertion site. Additionally, the device is equipped with a thread at the end for easy retraction after completed drug release. This device can release progesterone over a period
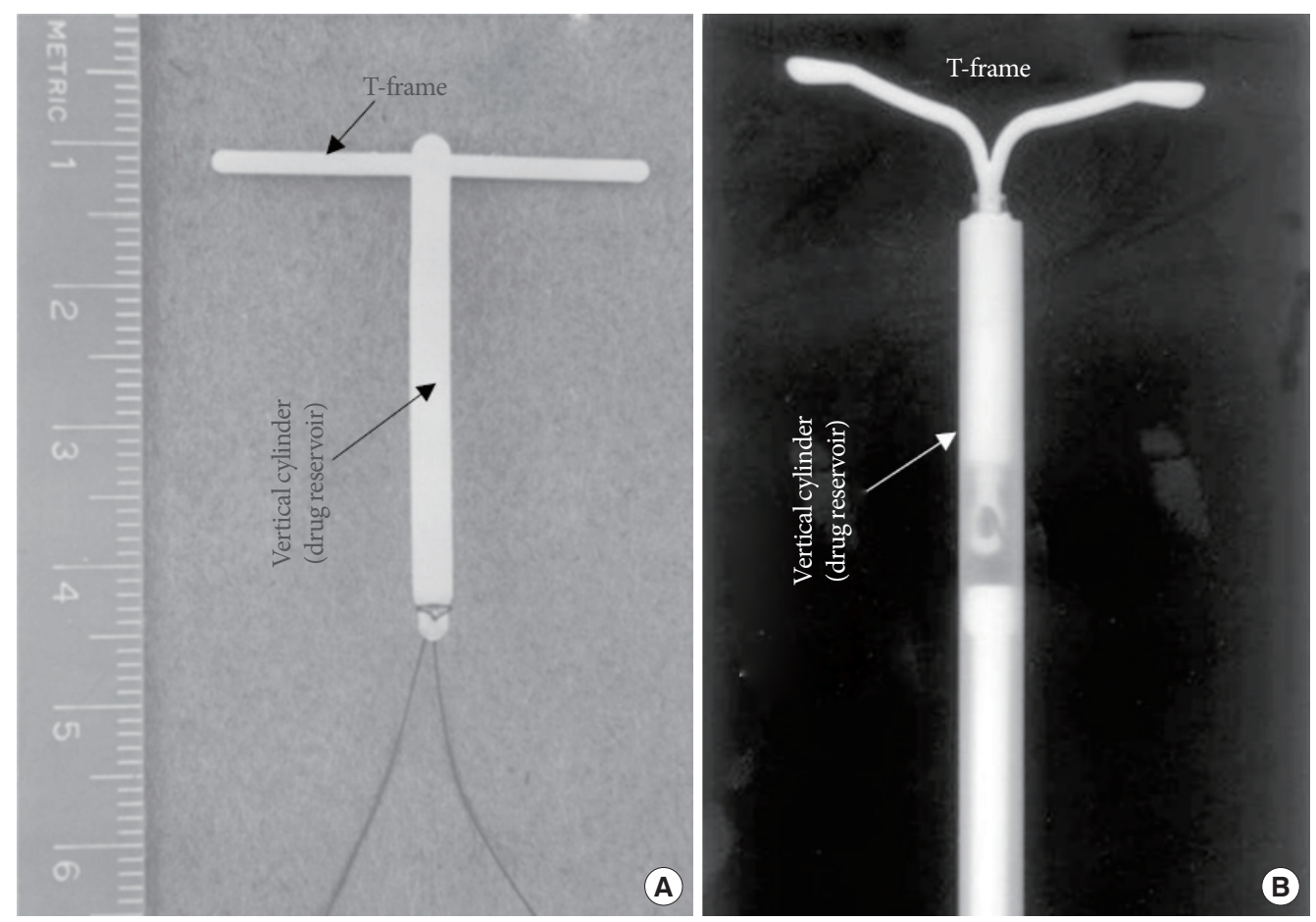

Fig. 2. T-shaped devices for intrauterine drug delivery. (A) Progestasert (Alza, Mountain View, CA, USA). The ruler is $6.5 \mathrm{~cm}$ long. Adapted from Wan LS, et al. Contraception 1977;16:417-34, with permission of Elsevier [16]. (B) Mirena (Bayer HealthCare Pharmaceuticals, Whippany, NJ, USA). Adapted from Zhou L, et al. Pharmacoepidemiol Drug Saf 2003;12:371-7, with permission of John Wiley \& Sons, Inc. [17]. 


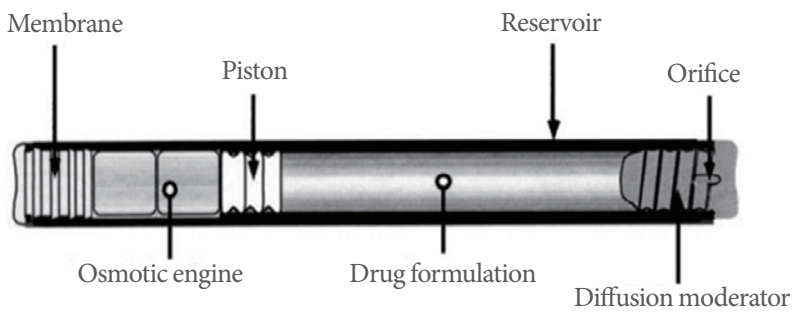

A

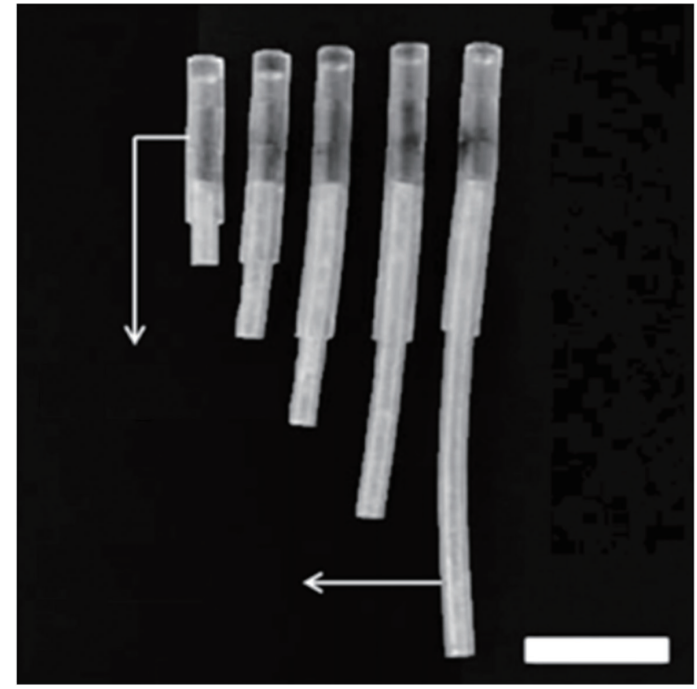

B

Fig. 3. Tube-shaped devices for highly controlled drug delivery. (A) Schematic diagram of the DUROS Implant (Alza, Mountain View, CA, USA). Adapted from Wright JC, et al. J Control Release 2001;75:1-10, with permission of Elsevier [19]. (B) Multiple tube assembly for controlled drug delivery. Adapted from Park M, et al. Macromol Res 2012;20: 960-7, with permission of Springer [22].

of 1 year after insertion due to the presence of a drug-diffusion barrier in the reservoir. Similarly, Mirena (Bayer HealthCare Pharmaceuticals, Whippany, NJ, USA) is also a T-shaped device for levonorgestrel delivery into the uterus (Fig. 2B). The vertical cylinder of the device is a drug reservoir made of $\mathrm{PE}$ and polydimethylsiloxane. The T-frame is made of $\mathrm{PE}$, which is also impregnated with barium sulfate to allow X-ray imaging for device localization. The release rate of the device is $20 \mu \mathrm{g} /$ day over a period of $\geq 5$ years [17].

For more controlled drug release, there are several tubeshaped devices under investigation, which were originally for local and systemic drug delivery [18]. The DUROS system by Alza was developed in 2000 for leuprolide delivery for the treatment of prostate cancer (Fig. 3A). A notable characteristic of this device is its ability to release drug at a constant rate. The
DUROS is shaped as a cylinder (45 $\mathrm{mm}$ long and $4 \mathrm{~mm}$ in diameter), in which a rate-controlling semipermeable membrane, an osmotic engine containing $\mathrm{NaCl}$, an elastomeric piston, a drug-containing reservoir, a diffusion moderator and an orifice are formed in series. Thus, when the device is implanted subcutaneously, the aqueous fluid is absorbed via the semipermeable membrane to expand the osmotic materials, which in turn, displaces the piston to push the drug via the diffusion mediator and orifice. Because the drug is pumped rather than diffused, a predetermined, constant amount of drug can be released per unit time $[19,20]$.

For better control of drug release, a precisely sized orifice was added to the tube-shaped devices [21]. This device is rather simple in structure: the tube is seamlessly filled with drug formulation, and in the tube, a precise microhole is fabricated as conduit for drug release. With this approach, the drug release rate can be modulated to be almost constant, according to the Fick's law and the estimated diffusion flux through a precisely fabricated microhole. In addition, further tailoring drug release was achieved relatively easily by controlling the number of microholes and total volume of the tube and by varying the material composition of the drug formulation in the tube. The prototype device was made of a polyimide tube $(20 \mathrm{~mm}$ in length, $1.8 \mathrm{~mm}$ in inner diameter), which was loaded with a model compound, fluorescein mixed with stearic acid (40:60), to test the feasibility. The results revealed that the drug release rate could be precisely controlled via a single microhole, $150 \mu \mathrm{m}$ in diameter, fabricated in the middle of the tube. When subcutaneously implanted in living mice, the device was released fluorescein continuously for 84 days.

To better program drug release scenarios, a device assembled with multiple distinct tubes was suggested (Fig. 3B) [22]. In this work, a silicone tube was first filled with a model compound to be delivered (i.e., fluorescein) to prepare a drug tube, and another silicone tube was filled with a water-soluble biocompatible polymer, polyethylene oxide to prepare a diffusion-barrier tube. Thus, a drug-delivery tube could be prepared by assembling a drug and diffusion-barrier tube in series, and the drug release could be modulated by the length of the diffusion-barrier tube. Moreover, drug-delivery tubes, each equipped with a diffusion-barrier tube of different length, could be combined for further assembly and this strategy was shown to allow continuous or even pulsatile drug release while maintaining an overall tube shape. 


\section{CONCLUSIONS}

The relatively low patient compliance of the conventional therapy of repeated intravesical drug instillation is considered an unmet clinical need for the treatment of bladder diseases. To resolve this, implantable devices enabled with sustained intravesical drug delivery have been studied. For easy insertion via the urinary tract, the devices are designed to be mostly tubeshaped, and their shape changes to a more complicating shape to allow device retention in the bladder. More importantly, to allow sustained drug release, the devices include a drug reservoir with a properly formed barrier for drug diffusion. Given such design criteria, there are many candidate devices with tube-like shape that are already actively studied. Those devices allow more precise, accurate control of drug release. Therefore, albeit currently used for drug delivery to the other target sites of interest, such novel devices are applicable to intravesical drug delivery.

\section{REFERENCES}

1. Macdiarmid SA, Sand PK. Diagnosis of interstitial cystitis/ painful bladder syndrome in patients with overactive bladder symptoms. Rev Urol 2007;9:9-16.

2. Edmondson SD, Zhu C, Kar NF, Di Salvo J, Nagabukuro H, SacreSalem B, et al. Discovery of vibegron: a potent and selective $\beta 3$ adrenergic receptor agonist for the treatment of overactive bladder. J Med Chem 2016;59:609-23.

3. Argade S, Chermansky C, Tyagi P. Biomarkers for interstitial cystitis/painful bladder syndrome. Womens Health (Lond Engl) 2016;12:87-90.

4. Rovner ES, Wein AJ. Once-daily, extended-release formulations of antimuscarinic agents in the treatment of overactive bladder: a review. Eur Urol 2002;41:6-14.

5. Diokno AC, Appell RA, Sand PK, Dmochowski RR, Gburek BM, Klimberg IW, et al. Prospective, randomized, double-blind study of the efficacy and tolerability of the extended-release formulations of oxybutynin and tolterodine for overactive bladder: results of the OPERA trial. Mayo Clin Proc 2003;78:687-95.

6. Lose G, Norgaard JP. Intravesical oxybutynin for treating incontinence resulting from an overactive detrusor. BJU Int 2001;87:76773.

7. Chuang YC, Lee WC, Lee WC, Chiang PH. Intravesical liposome versus oral pentosan polysulfate for interstitial cystitis/painful bladder syndrome. J Urol 2009;182:1393-400.
8. Parsons CL. Successful downregulation of bladder sensory nerves with combination of heparin and alkalinized lidocaine in patients with interstitial cystitis. Urology 2005;65:45-8.

9. Oosterlinck W, Kurth KH, Schroder F, Bultinck J, Hammond B, Sylvester R. A prospective European Organization for Research and Treatment of Cancer Genitourinary Group randomized trial comparing transurethral resection followed by a single intravesical instillation of epirubicin or water in single stage Ta, T1 papillary carcinoma of the bladder. J Urol 1993;149:749-52.

10. Nickel JC, Moldwin R, Lee S, Davis EL, Henry RA, Wyllie MG. Intravesical alkalinized lidocaine (PSD597) offers sustained relief from symptoms of interstitial cystitis and painful bladder syndrome. BJU Int 2009;103:910-8.

11. Lazzeri M, Spinelli M, Beneforti P, Malaguti S, Giardiello G, Turini D. Intravesical infusion of resiniferatoxin by a temporary in situ drug delivery system to treat interstitial cystitis: a pilot study. Eur Urol 2004;45:98-102.

12. Fraser MO, Lavelle JP, Sacks MS, Chancellor MB. The future of bladder control-intravesical drug delivery, a pinch of pepper, and gene therapy. Rev Urol 2002;4:1-11.

13. Herbison P, Hay-Smith J, Ellis G, Moore K. Effectiveness of anticholinergic drugs compared with placebo in the treatment of overactive bladder: systematic review. BMJ 2003;326:841-4.

14. Lee H, Cima MJ. An intravesical device for the sustained delivery of lidocaine to the bladder. J Control Release 2011;149:133-9.

15. Nickel JC, Jain P, Shore N, Anderson J, Giesing D, Lee H, et al. Continuous intravesical lidocaine treatment for interstitial cystitis/ bladder pain syndrome: safety and efficacy of a new drug delivery device. Sci Transl Med 2012;4:143ra100.

16. Wan LS, Hsu YC, Ganguly M, Bigelow B. Effects of the Progestasert on the menstrual pattern, ovarian steroids and endometrium. Contraception 1977;16:417-34.

17. Zhou L, Harrison-Woolrych M, Coulter DM. Use of the New Zealand Intensive Medicines Monitoring Programme to study the levonorgestrel-releasing intrauterine device (Mirena). Pharmacoepidemiol Drug Saf 2003;12:371-7.

18. Kleiner LW, Wright JC, Wang Y. Evolution of implantable and insertable drug delivery systems. J Control Release 2014;181:1-10.

19. Wright JC, Tao Leonard S, Stevenson CL, Beck JC, Chen G, Jao $\mathrm{RM}$, et al. An in vivo/in vitro comparison with a leuprolide osmotic implant for the treatment of prostate cancer. J Control Release 2001;75:1-10

20. Herrlich S, Spieth S, Messner S, Zengerle R. Osmotic micropumps for drug delivery. Adv Drug Deliv Rev 2012;64:1617-27.

21. Rastogi A, Bowman PD, Stavchansky S. Evaluation of a perforated 


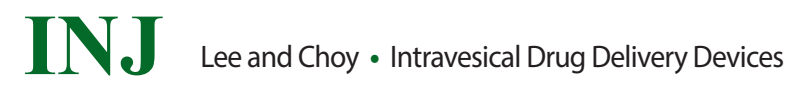

drug delivery system in mice for prolonged and constant release of a hydrophilic drug. Drug Deliv Transl Res 2012;2:106-11.

22. Park M, Park CG, Lee SH, Lee JE, Cho E, Prausnitz MR, et al. Poly- meric tube-shaped devices with controlled geometry for programmed drug delivery. Macromol Res 2012;20:960-7. 